ECCLRC

\title{
Multilevel hybrid spectral element ordering algorithms
}

Jennifer A. Scott

May 2004 
(C) Council for the Central Laboratory of the Research Councils

Enquires about copyright, reproduction and requests for additional copies of this report should be addressed to:

Library and Information Services

CCLRC Rutherford Appleton Laboratory

Chilton Didcot

Oxfordshire OX11 OQX

UK

Tel: $+44(0) 1235445384$

Fax: $+44(0) 1235446403$

Email: library@rl.ac.uk

CCLRC reports are available online at:

http://www.clrc.ac.uk/Activity/ACTIVITY=Publications;SECTION=225;

ISSN 1358-6254

Neither the Council nor the Laboratory accept any responsibility for loss or damage arising from the use of information contained in any of their reports or in any communication about their tests or investigations. 


\title{
Multilevel hybrid spectral element ordering algorithms $\mathbf{s}^{1,2}$
}

\author{
Jennifer A. Scott
}

\begin{abstract}
For frontal solvers to perform well on finite-element problems it is essential that the elements are ordered for a small wavefront. Multilevel element ordering algorithms have their origins in the profile reduction algorithm of Sloan but for large problems often give significantly smaller wavefronts. We examine a number of multilevel variants with the aim of finding the best methods to include within a new state-of-the-art frontal solver for finite-element applications that we are currently developing. Numerical experiments are performed using a range of problems arising from real applications and comparisons are made with existing element ordering algorithms.
\end{abstract}

Keywords: large sparse linear systems, finite elements, element ordering, frontal method.

\footnotetext{
${ }^{1}$ Current reports available from "http://www.numerical.rl.ac.uk/reports/reports.html".

2 This work was supported by the EPSRC grant GR/S42170.
}

Computational Science and Engineering Department

Atlas Centre

Rutherford Appleton Laboratory

Oxon OX11 0QX

May 2004. 


\section{Introduction}

The frontal method is frequently the method of choice for solving the large sparse systems of linear equations that arise during the solution of finite-element problems. These systems are of the form

$$
A X=B
$$

where the $n \times n$ matrix $A$ is a sum of nelt finite-element matrices

$$
A=\sum_{l=1}^{n e l t} A^{(l)},
$$

and $B$ is an $n \times n r h s$ matrix $(n r h s \geq 1)$ of known right-hand sides. Each matrix $A^{(l)}$ has nonzeros only in a few rows and columns; $A^{(l)}$ corresponds to the contribution from element $l$ and is normally held as a small dense matrix. One reason for choosing a frontal method is that only a small amount of main memory is required to solve the problem. This allows very much larger problems to be solved than is possible using a direct solver that works entirely incore. However, this is only true if it is possible to preorder the elements to ensure small fronts throughout the computation. If $a_{i j}$ and $a_{i j}^{(l)}$ denote the $(i, j)$ th entry of $A$ and $A^{(l)}$, respectively, the basic assembly operation for constructing $A$ is of the form

$$
a_{i j} \Leftarrow a_{i j}+a_{i j}^{(l)}
$$

The main feature of the frontal method is that the Gaussian elimination operation

$$
a_{i j} \Leftarrow a_{i j}-a_{i l}\left[a_{l l}\right]^{-1} a_{l j}
$$

may be performed once all the terms in the triple product in (1.4) are fully summed. A variable is fully summed if it is involved in no further sums of the form (1.3) and is partially summed if it has appeared in at least one of the elements assembled so far but is not yet fully summed. Thus by assembling the contributions $A^{(l)}$ from the finite-elements one at a time and (provided numerical stability conditions are satisfied) performing eliminations as variables become fully summed, the construction of the assembled coefficient matrix $A$ is avoided.

At each stage of the assembly and elimination processes, the fully and partially summed variables are held in an in-core frontal matrix. In the innermost loop of the numerical factorization, dense linear algebra operations are performed on the frontal matrix. For efficiency, in terms of both storage and arithmetic operations, the elements must be assembled in an order that keeps the size of the frontal matrix, known as the wavefront, as small as possible. In other words, the elements need to be ordered so that partially summed variables become fully summed as soon as possible. Of interest is: 
- the maximum wavefront, since this affects the in-core storage needed,

- the sum of the wavefronts, known as the profile, since this determines the total storage needed for the matrix factors, and

- the root-mean-square wavefront, since the work performed when eliminating a variable is proportional to the square of the current wavefront.

Reflecting the popularity of the frontal method, a number of algorithms for automatically ordering finite elements for small wavefront and profile have been reported on in the literature (see, for example, Scott, 1999 for references to element ordering algorithms). Duff, Reid and Scott (1989) divide element ordering algorithms into direct and indirect algorithms. As the name suggests, direct algorithms order the elements directly while indirect algorithms use a two-step approach in which the variables are first relabelled and then used to resequence the elements; the new variable indices are subsequently discarded. Duff et al. report that both approaches can be used effectively and in their experiments neither was found to be consistently superior to the other.

Some of the most well-known element ordering algorithms are based on the profile and wavefront reduction algorithm of Sloan (1986). The Sloan algorithm exploits the close relationship between a matrix $A$ of order $n$ with a symmetric sparsity pattern and its undirected graph with $n$ nodes, that is, the adjacency graph $\mathcal{G}(A)$. In particular, it uses the level set structure of $\mathcal{G}(A)$. In the late 1990s, Scott (1999) developed an element ordering package MC63 for inclusion in the HSL mathematical software library (HSL, 2004). This package provides efficient implementations of a number of variants of Sloan's algorithm. In particular, it offers a hybrid variant in which Sloan's algorithm is used to refine an ordering provided by the user. Numerical results reported by Scott (1999) showed that if the user inputs a spectral ordering then, for large problems, the hybrid method is a significant improvement on Sloan's algorithm (that is, it generally produces smaller maximum and root-mean-squared wavefronts). The disadvantage of the hybrid spectral-Sloan algorithm is the need to compute a spectral ordering, which can add significantly to the overall cost of ordering the elements.

Spectral orderings are expensive because they are dependent upon the computation of the eigenvector corresponding to the smallest non-trivial eigenvalue of the Laplacian matrix associated with the graph of the problem, the so-called Fiedler vector (Fiedler, 1975). Recently, a new flexible software package that implements both an efficient multilevel algorithm for computing the Fiedler vector and a number of multilevel profile reduction algorithms has been designed and developed by $\mathrm{Hu}$ and Scott (2003). The new Fortran 95 code is called HSL_MC73 and will be included in HSL (2004). The aim of this article is to report on using HSL MC73 to obtain high quality multilevel element orderings efficiently for use with a frontal solver.

The outline of this report is as follows. In Section 2, the new code HSL_MC73 is briefly described then, in Section 3, we look at how we can use HSL_MC73 to obtain a number of multilevel element ordering algorithms. Both direct and indirect variants will be proposed. Numerical results are presented for the multilevel algorithms in Section 4. Finally, some concluding remarks are made in Section 5. 


\section{A new multilevel profile reduction code}

We start by briefly describing the new multilevel Fiedler and profile reduction code HSL _MC73.

Following the success of spectral orderings for graph partitioning, Barnard, Pothen and Simon (1995) first proposed using the Fiedler vector to obtain profile reducing orderings for matrices $A$ with symmetric sparsity patterns. Their algorithm is motivated as an attempt to minimize the two-sum

$$
\min _{\mathbf{x} \in \mathcal{P}}\left\{\sum_{\left\{i<j: a_{i j} \neq 0\right\}}\left(x_{i}-x_{j}\right)^{2}\right\},
$$

where $\mathcal{P}$ denotes the set of vectors whose components are permutations of

$$
i-(n+1) / 2, \quad i=1,2, \ldots, n \text {. }
$$

That is,

$$
\min _{\mathbf{x} \in \mathcal{P}} \mathbf{x}^{T} L \mathbf{x}
$$

where $L$ is the Laplacian of $A$ given by

$$
L=\left\{l_{i j}\right\}= \begin{cases}-1 & \text { if } i \neq j \text { and } \quad a_{i j} \neq 0 \\ 0 & \text { if } i \neq j \text { and } \quad a_{i j}=0 \\ \sum_{i \neq j}\left|l_{i j}\right| & \text { if } i=j .\end{cases}
$$

To make this problem tractable, albeit at the expense of not computing a guaranteed optimal solution, a heuristic is introduced. Instead of minimizing over the discrete set $\mathcal{P}$, the problem (2.6) is relaxed to $\mathbf{x} \in \mathcal{R}^{n}$ with $\mathbf{x}^{T} \mathbf{e}=0\left(\mathbf{e}=[1,1, \ldots, 1]^{T}\right)$ and $\|\mathbf{x}\|_{2}=\|\mathbf{p}\|_{2}$ for any $\mathbf{p} \in \mathcal{P}$. The solution is then the eigenvector corresponding to the second smallest eigenvalue of $L$, that is, the Fiedler vector. Applying the permutation induced by ordering the components of this vector into monotonic order to the matrix $A$ gives the so-called spectral ordering. In general, it not only reduces the two-sum but also the profile and wavefront of $A$.

The main problem with implementing the spectral method is that computing eigenvectors of large matrices is expensive. This led Barnard and Simon (1994) to propose a multilevel algorithm for computing the Fiedler vector. The basic multilevel Fiedler algorithm proceeds as follows:

- Starting with the adjacency graph $\mathcal{G}(A)$, a series of graphs of successively coarser (smaller) sizes is generated.

- At some point the graph has so few nodes that it is very cheap to compute the Fiedler vector of the associated Laplacian.

- The coarse graph Fiedler vector is projected from one level to another. At each level some refinement is performed until, finally, an (approximate) Fiedler vector for the original Laplacian is obtained.

In broad terms, this is the algorithm that is implemented within the new software package HSL_MC73. A key observation is that, for both graph partitioning and for profile reduction algorithms, it is not necessary to obtain the Fiedler vector to high accuracy; instead an approximate Fiedler vector is sufficient. Thus HSL_MC73 is designed to compute an approximate Fiedler vector and a number of parameters under the user's control are used in determining how 
accurate the requested eigenvector is. Full details of the algorithm, its implementation and user interface are given in $\mathrm{Hu}$ and Scott (2003).

As well as offering a multilevel spectral ordering algorithm for profile reduction, HSL_MC73 includes implementations of the hybrid Sloan algorithm of Kumfert and Pothen (1997) and the multilevel Sloan algorithm of $\mathrm{Hu}$ and Scott (2001). The Sloan algorithm for profile and wavefront reduction employs the adjacency graph $\mathcal{G}(A)$ of $A$ and has two distinct phases:

1. Selection of a start node $s$ and a target end node $e$.

2. Node reordering.

The first phase computes a pseudodiameter of $\mathcal{G}(A)$ and uses it to provide $s$ and $e$. In the second phase, the chosen start node is numbered first and a list of nodes that are eligible to be numbered next is formed. At each stage of the numbering, the list of eligible nodes comprises the neighbours of the nodes that have already been renumbered together with their neighbours. The next node to be numbered is selected from the list of eligible nodes to maximise the priority function

$$
P(i)=-W_{1} \operatorname{inc}(i)+W_{2} \operatorname{dist}(i, e),
$$

where $\left(W_{1}, W_{2}\right)$ are fixed positive weights. The first term, inc $(i)$, is the amount by which the wavefront will increase if node $i$ is ordered next. The second term, $\operatorname{dist}(i, e)$, is the distance between nodes $i$ and the end node $e$. Thus, a balance is maintained between the aim of keeping the wavefront small and bringing in nodes that have been left behind (that is, those far away from the target end node $e$ ). A node has a high priority if it causes either no increase or only a small increase to the current wavefront size and is at a large distance from the end node $e$.

Kumfert and Pothen (1997) observed that there are problems on which the spectral algorithm can perform poorly and this motivated them to propose a hybrid method that combines use of the spectral ordering with a modified version of the second phase of Sloan's algorithm. The first term in (2.8) affects the priority function in a local way, by giving higher priority to nodes that will result in a small (or negative) increase to the current wavefront. This is done in a greedy fashion, without consideration of the long-term effect. The second term acts in a more global manner, ensuring nodes lying far away from the end node are not left behind. The second phase of the Sloan algorithm can therefore be viewed as an algorithm that refines the ordering implied by the distance function dist $(i, e)$. Thus Kumfert and Pothen modified the second phase of Sloan's algorithm so that, in place of the distance function, it refined the spectral ordering. Their numerical experiments showed that, for large problems, the resulting hybrid method generally gives significantly smaller profiles than those obtained using the standard Sloan algorithm. This led us to design the package HSL_MC73 to include an option to compute a multilevel spectral ordering which is then refined to obtain the so-called hybrid spectral-Sloan ordering (for further details, see Reid and Scott, 1999 and $\mathrm{Hu}$ and Scott, 2003).

The main disadvantage of the hybrid profile reduction method is that it requires significantly more CPU time than Sloan's algorithm because it is more expensive to compute the Fiedler vector than it is to find a pseudodiameter for $A$ using the (modified) Gibbs-Poole-Stockmeyer algorithm of Reid and Scott (1999). Even if the Fiedler vector is computed as in HSL_MC73 using a multilevel approach, the hybrid algorithm can be relatively expensive. In an attempt to avoid computation of the Fiedler vector while still maintaining the quality of the hybrid algorithm, $\mathrm{Hu}$ and Scott (2001) proposed a multilevel version of Sloan's algorithm. Mirroring the multilevel Fiedler algorithm, the multilevel Sloan profile reduction algorithm comprises three separate steps: 
- A series of graphs of successively smaller sizes is generated.

- The coarsest graph is reordered using the Sloan algorithm.

- The coarse graph ordering is projected from one level to another by first mapping the ordering for the previous (coarser) level onto the current level and then performing refinement using the second phase of Sloan's algorithm.

Numerical results presented by $\mathrm{Hu}$ and Scott confirm that this approach is faster than the hybrid method and, with appropriate coarsening and refinement, produces orderings that are of comparable quality. Thus, in addition to the multilevel spectral and hybrid methods, HSL _MC73 includes an efficient implementation of the multilevel Sloan algorithm for profile reduction.

\section{Multilevel element orderings}

The input required by the package HSL MC73 is the sparsity pattern of the matrix $A$ or, equivalently, the adjacency graph $\mathcal{G}(A)$. In fact, any undirected (unweighted) graph can be input and we use this facility to obtain multilevel element ordering algorithms.

There are a number of possible graphs associated with a finite-element problem that have been used for element resequencing. We consider two that our previous experiments (Duff et al., 1989 and Scott, 1999) found to be efficient with the Sloan algorithm: the supervariable connectivity graph and the element communication graph. We will input these graphs to HSL MC73 and use them to obtain multilevel indirect and direct element orderings, respectively.

In the variable connectivity graph the nodes are the variables defined on the finite-element mesh, and the edges are constructed by making the variables of each element pairwise adjacent. This graph is the adjacency graph $\mathcal{G}(A)$ of the assembled finite-element matrix $A$. However, because in many finite-element problems there are a number of degrees of freedom at each node of the finite-element mesh, a more compact representation of the finite-element problem is generally possible through the use of supervariables. A supervariable is a collection of one or more variables, such that each variable belongs to the same set of finite elements. The finite-element mesh can be transformed into a supervariable connectivity graph $\mathcal{G}_{S}$, whose nodes are the supervariables and whose edges are formed by making the supervariables of each finite element pairwise adjacent.

For finite-element problems, element connectivity graphs may be defined in which the nodes are the finite elements. There is more than one way in which the element connectivity may then be defined. We restrict our attention to the element communication graph $\mathcal{G}_{E C}$ in which two elements are defined as being adjacent whenever they share at least one variable in common.

\subsection{Indirect multilevel element orderings}

Our indirect multilevel element ordering algorithms proceed as follows:

- Generate the supervariable graph $\mathcal{G}_{S}$ of $A$.

- Apply HSL_MC73 to $\mathcal{G}_{S}$ to obtain an ordering of the supervariables.

- Order the elements in ascending sequence of their lowest numbered supervariable and then discard the supervariable ordering. 
When HSL_MC73 is applied to $\mathcal{G}_{S}$ we have two options. We can either use the multilevel Sloan algorithm or the hybrid spectral-Sloan algorithm (which employs the multilevel Fiedler algorithm). We have performed experiments with both approaches and include results in Section 4. We can also try and improve the HSL_MC73 supervariable ordering prior to resequencing the elements using the Hager exchange algorithms. Hager (2002) suggested two methods for improving any given profile reducing permutation of a symmetric matrix $A$; a down exchange algorithm and an up exchange algorithm, which he proposed using in an iterative fashion (further details and results illustrating the effectiveness of the exchanges are given in Reid and Scott, 2002). HSL_MC73 includes an option to perform a user-chosen number of down/up exchanges; we include results in Section 4 for using this option within our indirect multilevel element ordering algorithm.

\subsection{Direct multilevel element orderings}

Using an analogous approach, our direct multilevel element ordering algorithms comprise the following steps:

- Generate the element communication graph $\mathcal{G}_{E C}$ of $A$.

- Apply HSL_MC73 to $\mathcal{G}_{E C}$ to obtain either a multilevel spectral ordering or a multilevel Sloan ordering for $\mathcal{G}_{E C}$.

- Refine the element ordering using a modified version of the second step of Sloan's algorithm.

The modified version of Sloan's algorithm that we use to refine the element ordering computed by $\mathcal{G}_{E C}$ is described in detail in Scott (1999) and is implemented in the HSL package MC63. Again, Hager exchanges may be used when ordering $\mathcal{G}_{E C}$ using HSL_MC73.

\section{Numerical results}

The numerical experiments reported on in this section were performed on a single Xeon $3.06 \mathrm{GHz}$ processor of a Dell Precision Workstation 650 with 4 GBytes of RAM under the Fedora Core 1 Linux operating system. The NAG Fortran 95 compiler was used with the compiler optimization flag -0. All timings are CPU times, measured using the Fortran 95 routine cpu_time and are given in seconds. Unless otherwise stated, the default values are used for all MC63 and HSL_MC73 control parameters.

The test problems used in our numerical experiments are listed in Table 4.1. The problems range in size from fewer than 1,000 elements to more than 70,000 elements with almost 225,000 degrees of freedom. For each problem the order $n$ of $A$ together with the number nsup of supervariables is given. For cham and tubu, only lists of supervariables belonging to each element were available so for these problems $n=n$ sup. For the remaining problems, we note that the number of supervariables is significantly less than the number of variables.

\subsection{Use of supervariables}

To illustrate the importance of using supervariables, in Table 4.2 we present results for the indirect multilevel Sloan algorithm using the variable connectivity graph $\mathcal{G}(A)$ and the supervariable 
Table 4.1: The test problems. $n$ and nsup denote the number of variables and supervariables, respectively.

\begin{tabular}{lrrrl}
\hline \hline & & & & \\
Identifier & $n$ & $n s u p$ & Elements & Description/discipline \\
& & & & \\
\hline cham & 12834 & 12834 & 11070 & Part of an engine cylinder \\
crplat2 & 18010 & 3004 & 3152 & Corrugated plate field \\
fcondp2 & 201822 & 33913 & 35836 & Oil production platform \\
fullb & 199187 & 33442 & 59738 & Full-breadth barge \\
halfb & 224617 & 38556 & 70211 & Half-breadth barge \\
inv-ext-2 & 78142 & 19734 & 7193 & Fluid flow \\
mt1 & 97578 & 17044 & 5328 & Tubular joint \\
opt1 & 15449 & 3802 & 977 & Part of condeep cylinder \\
ship_001 & 34920 & 5843 & 3431 & Ship structure - predesign \\
ship_003 & 121728 & 20287 & 45464 & Ship structure - production \\
shipsec1 & 140874 & 23479 & 41037 & Section of a ship \\
shipsec5 & 179860 & 17260 & 52272 & Section of a ship \\
shipsec8 & 114919 & 19532 & 32580 & Section of a ship \\
srb1 & 54924 & 9154 & 9240 & Space shuttle rocket booster \\
thread & 29736 & 8838 & 2176 & Threaded connector \\
trdheim & 22098 & 2868 & 813 & Mesh of the Trondheim fjord \\
troll & 213453 & 48435 & 41084 & Structural analysis \\
tsyl201 & 20685 & 2881 & 960 & Part of condeep cylinder \\
tubu & 26573 & 26573 & 23446 & Engine cylinder model \\
x104 & 108384 & 17260 & 26019 & Beam joint \\
\hline \hline
\end{tabular}

graph $\mathcal{G}_{S}$. We see that using supervariables leads to large savings in the time required to reorder the elements. For most of our examples, the time is reduced by a factor of more than 10 . Furthermore, for many of the problems, the root-mean-square wavefront is significantly smaller if supervariables are used. It appears that the initial coarsening of the variable connectivity graph by using supervariables is generally more effective than the coarsening used within the multilevel code HSL_MC73. Note that, although not given here, the root-mean-square wavefronts for the direct multilevel element ordering algorithms are not affected by using supervariables in place of variables but there is a small time saving if supervariables are used. All results in the remainder of this report are computed using supervariables.

\subsection{Sloan vs multilevel algorithms}

In Table 4.3 we compare the performance of the Sloan algorithm (as implemented within MC63) with the multilevel algorithms. We include both direct and indirect variants. For the multilevel algorithms, we report results for the hybrid spectral-Sloan algorithm (with the spectral ordering computed using HSL_MC73) and for the multilevel Sloan algorithm (again computed using HSL_MC73 and, for the direct algorithm, refined using MC63). For each problem, the smallest rootmean-square wavefront (and any within 5 per cent of the smallest) are highlighted in bold. Note that when assessing the relative performance of the algorithms we make no distinction between 
Table 4.2: The root-mean-square wavefronts and times required by the indirect multilevel Sloan algorithm using the variable and supervariable connectivity graphs.

\begin{tabular}{lrrrc}
\hline \hline Identifier & \multicolumn{2}{c}{ r.m.s } & \multicolumn{2}{c}{ time } \\
& $\mathcal{G}(A)$ & \multicolumn{1}{c}{$\mathcal{G}_{S}$} & $\mathcal{G}(A)$ & $\mathcal{G}_{S}$ \\
\hline crplat2 & 332 & 260 & 0.24 & 0.01 \\
fcondp2 & 2631 & 1862 & 3.25 & 0.23 \\
fullb & 1943 & 3110 & 3.32 & 0.33 \\
halfb & 1638 & 1462 & 3.49 & 0.35 \\
inv-ext-2 & 3378 & 2272 & 9.18 & 0.37 \\
mt1 & 1339 & 1626 & 2.37 & 0.15 \\
opt1 & 530 & 526 & 0.44 & 0.04 \\
ship_001 & 460 & 450 & 1.57 & 0.05 \\
ship_003 & 1400 & 1544 & 3.28 & 0.22 \\
shipsec1 & 2398 & 1686 & 2.51 & 0.22 \\
shipsec5 & 1496 & 1370 & 2.80 & 0.28 \\
shipsec8 & 2377 & 1701 & 1.86 & 0.19 \\
srb1 & 318 & 327 & 0.71 & 0.05 \\
thread & 2294 & 1706 & 1.06 & 0.13 \\
trdheim & 145 & 161 & 0.42 & 0.02 \\
troll & 4265 & 2377 & 3.71 & 0.46 \\
tsyl201 & 505 & 513 & 0.57 & 0.02 \\
x104 & 1020 & 1106 & 3.56 & 0.11 \\
\hline \hline
\end{tabular}

the smallest wavefront and those that are close to the smallest since tie-breaking strategies within the implementation of each algorithm can lead to small variations in the computed wavefronts. Looking first at the results for the direct ordering algorithms (that is, the numbers in columns 2, 4 and 6), we see that both the hybrid spectral-Sloan and the multilevel Sloan algorithms are generally an improvement on the Sloan algorithm. For many of the larger problems, including fcondp2 and shipsec1, the improvements are substantial. Comparing the two direct multilevel variants, the direct multilevel Sloan algorithm does not perform as well as the direct hybrid spectral-Sloan. Similar conclusions can be drawn when comparing the different indirect variants. Overall, the best method appears to be the indirect hybrid spectral-Sloan algorithm, with the advantage over the MC63 Sloan algorithm being greatest for the large test problems (those with more than about 10,000 elements). The indirect hybrid algorithm produces the best (or close to the best) results for the majority of our test problems.

If only a single or small number of matrix factorizations and solves are to be performed following the reordering of the elements, the cost of reordering the elements may be a concern. Timings for a subset of our test problems are given in Table 4.4. The Sloan algorithm (MC63) is clearly significantly faster than the multilevel and hybrid variants, particularly for the problems with a large number of supervariables and elements. As already noted, $\mathrm{Hu}$ and Scott (2001) introduced the multilevel Sloan profile reduction algorithm to save on the time required to compute a spectral ordering and we do achieve some savings in the element ordering times by using the multilevel Sloan algorithm rather than the spectral approach. For our examples, the indirect multilevel algorithm is between 25 and 50 per cent faster than the indirect hybrid 
Table 4.3: The root-mean-square wavefronts computed by the Sloan and multilevel algorithms. * indicates the wavefront is larger than for the original ordering.

\begin{tabular}{lccrrrr}
\hline \hline Identifier & \multicolumn{2}{c}{ Sloan } & \multicolumn{2}{c}{ Hybrid } & \multicolumn{2}{c}{ Multilevel } \\
& \multicolumn{2}{c}{ (MC63) } & \multicolumn{2}{c}{ spectral-Sloan } & \multicolumn{2}{c}{ Sloan } \\
& direct & indirect & direct & indirect & direct & indirect \\
\hline cham & $\mathbf{3 3 2}$ & $\mathbf{3 3 2}$ & $\mathbf{3 3 4}$ & $\mathbf{3 3 2}$ & 368 & 691 \\
crplat2 & 334 & 327 & $\mathbf{2 3 4}$ & $\mathbf{2 4 4}$ & 271 & 257 \\
fcondp2 & 3024 & 2667 & 1827 & $\mathbf{1 7 0 0}$ & 2164 & 1863 \\
fullb & 2172 & 2021 & $\mathbf{1 8 7 9}$ & $\mathbf{1 8 3 3}$ & 2152 & 3110 \\
halfb & 1776 & 1608 & $\mathbf{1 4 1 1}$ & $\mathbf{1 3 6 5}$ & 1500 & 1462 \\
inv-ext-2 & $8429^{*}$ & $3379^{*}$ & $8632^{*}$ & $\mathbf{2 2 7 2}$ & $8699^{*}$ & $\mathbf{2 2 7 2}$ \\
mt1 & 1546 & 1335 & $\mathbf{1 0 1 8}$ & 1176 & 1149 & 1626 \\
opt1 & 619 & $\mathbf{5 2 8}$ & 539 & 557 & 592 & $\mathbf{5 2 6}$ \\
ship_001 & 693 & $\mathbf{4 5 1}$ & 500 & $\mathbf{4 6 1}$ & 510 & $\mathbf{4 5 0}$ \\
ship_003 & 1739 & 1558 & $\mathbf{1 4 2 7}$ & $\mathbf{1 3 7 1}$ & 1565 & 1544 \\
shipsec1 & 2629 & 2494 & 1895 & $\mathbf{1 4 4 4}$ & 1524 & 1686 \\
shipsec5 & 1803 & 1499 & $\mathbf{1 3 4 5}$ & $\mathbf{1 3 1 7}$ & 1425 & $\mathbf{1 3 7 0}$ \\
shipsec8 & 2080 & 2302 & 1746 & $\mathbf{1 6 4 4}$ & 1818 & 1701 \\
srb1 & $\mathbf{3 2 1}$ & $\mathbf{3 1 8}$ & $\mathbf{3 3 4}$ & $\mathbf{3 2 6}$ & 338 & $\mathbf{3 2 7}$ \\
thread & 2215 & 1962 & 1442 & $\mathbf{1 1 2 2}$ & 1948 & 1701 \\
trdheim & 172 & 146 & 147 & 155 & $\mathbf{1 3 5}$ & 161 \\
troll & $4334^{*}$ & $4229^{*}$ & 3912 & 3593 & 2669 & $\mathbf{2 3 7 7}$ \\
tsyl201 & $\mathbf{5 1 1}$ & $\mathbf{5 0 4}$ & $\mathbf{5 1 2}$ & $\mathbf{5 1 2}$ & $\mathbf{5 1 0}$ & $\mathbf{5 1 3}$ \\
tubu & $\mathbf{4 0 8}$ & 451 & $\mathbf{4 1 4}$ & $\mathbf{4 0 3}$ & 452 & 454 \\
x104 & 1064 & 1268 & $\mathbf{1 0 0 7}$ & 1061 & $\mathbf{9 8 9}$ & 1106 \\
\hline \hline
\end{tabular}

Table 4.4: The times (in seconds) for reordering the elements using the Sloan and multilevel algorithms.

\begin{tabular}{|c|c|c|c|c|c|c|}
\hline \multirow[t]{2}{*}{ Identifier } & \multicolumn{2}{|c|}{$\begin{array}{l}\text { Sloan } \\
\text { (MC63) }\end{array}$} & \multicolumn{2}{|c|}{$\begin{array}{c}\text { Hybrid } \\
\text { spectral-Sloan }\end{array}$} & \multicolumn{2}{|c|}{$\begin{array}{l}\text { Multilevel } \\
\text { Sloan }\end{array}$} \\
\hline & direct & indirect & direct & indirect & direct & indirect \\
\hline cham & 0.01 & 0.01 & 0.15 & 0.20 & 0.15 & 0.15 \\
\hline fcondp2 & 0.09 & 0.09 & 0.31 & 0.33 & 0.29 & 0.23 \\
\hline halfb & 0.20 & 0.13 & 0.79 & 0.44 & 0.73 & 0.35 \\
\hline $\mathrm{mt} 1$ & 0.02 & 0.05 & 0.06 & 0.22 & 0.05 & 0.15 \\
\hline ship_003 & 0.17 & 0.09 & 0.49 & 0.27 & 0.64 & 0.22 \\
\hline shipsec1 & 0.13 & 0.09 & 0.64 & 0.27 & 0.45 & 0.22 \\
\hline shipsec 8 & 0.13 & 0.08 & 0.44 & 0.25 & 0.44 & 0.19 \\
\hline thread & 0.01 & 0.04 & 0.03 & 0.23 & 0.03 & 0.13 \\
\hline x104 & 0.02 & 0.05 & 0.05 & 0.20 & 0.05 & 0.10 \\
\hline
\end{tabular}

algorithm. 


\subsection{Effect of Hager exchanges}

The results reported so far did not use Hager exchanges. The results in Table 4.5 illustrate the reductions in the root-mean-squared wavefront that are achieved by using Hager exchanges within the call to HSL_MC73. In these experiments, up to a maximum of 5 down/up exchanges were allowed (the number of exchanges performed is fewer than 5 if the reductions in the profile are less than a prescribed amount; see Reid and Scott, 2002 for details). Results are given for both the

Table 4.5: The root-mean-square wavefronts computed using the indirect spectral-Sloan and multilevel Sloan algorithms with and without Hager exchanges. Numbers in bold indicate a reduction of at least 5 per cent.

\begin{tabular}{lrrrr}
\hline \hline Identifier & \multicolumn{2}{c}{ Hybrid } & \multicolumn{2}{c}{ Multilevel } \\
& $\begin{array}{r}\text { spectral-Sloan } \\
\text { without }\end{array}$ & \multicolumn{1}{c}{ with } & without & \multicolumn{1}{c}{ with } \\
\hline cham & 332 & 330 & 691 & 680 \\
crplat2 & 244 & 232 & 257 & 239 \\
fcondp2 & 1700 & 1621 & 1863 & 1805 \\
fullb & 1833 & 1804 & 3110 & $\mathbf{2 8 3 2}$ \\
halfb & 1365 & 1362 & 1462 & $\mathbf{1 3 7 1}$ \\
ship_001 & 461 & 460 & 450 & 447 \\
ship_003 & 1371 & 1322 & 1544 & 1544 \\
shipsec1 & 1444 & 1418 & 1686 & $\mathbf{1 4 9 5}$ \\
shipsec5 & 1317 & 1276 & 1370 & $\mathbf{1 3 0 0}$ \\
shipsec8 & 1644 & $\mathbf{1 5 4 5}$ & 1701 & $\mathbf{1 5 6 2}$ \\
srb1 & 326 & 326 & 327 & 324 \\
trol1 & 3593 & $\mathbf{3 3 4 9}$ & 2377 & $\mathbf{2 2 4 9}$ \\
tubu & 403 & 383 & 454 & 444 \\
x104 & 1061 & 1052 & 1106 & 1104 \\
\hline \hline
\end{tabular}

indirect spectral-Sloan and multilevel Sloan algorithms. Problems for which the Hager exchanges do not reduce the root-mean-square wavefront are omitted while those for which the reduction exceeds 5 per cent are highlighted in bold. We see that there are only two problems for which the Hager exchanges applied to the hybrid spectral-Sloan ordering leads to a significant reduction in the wavefront. For the multilevel algorithm, Hager exchanges improve the ordering for a few more test examples and for some, including halfb and shipsec8, the multilevel plus Hager ordering is competitive with the hybrid spectral-Sloan ordering. However, using Hager exchanges can add a large overhead to the cost of the element ordering. For example, for problem halfb, the multilevel reordering time increases from 0.35 to 0.58 seconds and for shipsec 8 from 0.19 to 0.33 seconds.

\subsection{Element ordering with a frontal solver}

As already discussed, the main motivation behind the work in this report is the need to compute element orderings that are efficient when used with a frontal solver. In this section, we present results for using the best element ordering method that we have, that is the indirect hybrid spectral-Sloan algorithm, with the well-known HSL frontal solver MA42 of Duff and Scott (1996). 
Comparisons are made with ordering the elements for MA42 using MC63 (both the direct and indirect Sloan algorithms are run and for each problem the better of the two is selected). Numerical values in the range $(0,1)$ are generated for the entries of the matrices using the HSL pseudo random number generator routine FA14. Default settings are used for the MA42 control

Table 4.6: The results of using the hybrid spectral-Sloan ordering with the frontal solver MA42.

\begin{tabular}{lrrrrrr}
\hline Identifier & \multicolumn{2}{c}{ Time } & \multicolumn{2}{c}{$\begin{array}{c}\text { Number flops } \\
\left(* 10^{8}\right)\end{array}$} & \multicolumn{2}{c}{ Factor entries } \\
& (seconds) & $\left(* 10^{5}\right)$ & \\
& MC63 & Hybrid & MC63 & Hybrid & MC63 & Hybrid \\
\hline \hline cham & $\mathbf{3 . 1}$ & $\mathbf{3 . 2}$ & 29 & 29 & 85 & 85 \\
crplat2 & 4.0 & $\mathbf{2 . 3}$ & 40 & 20 & 118 & 84 \\
fcondp2 & 1657 & $\mathbf{7 8 5}$ & 27393 & 11560 & 9139 & 6100 \\
fullb & 1052 & $\mathbf{8 8 2}$ & 16117 & 13309 & 7735 & 7125 \\
halfb & 712 & $\mathbf{5 7 5}$ & 10463 & 8259 & 6477 & 5927 \\
inv-ext-2 & 962 & $\mathbf{4 7 8}$ & 16387 & 7631 & 4877 & 3249 \\
mt1 & 230 & $\mathbf{1 4 5}$ & 3462 & 2013 & 2397 & 1879 \\
opt1 & $\mathbf{7 . 2}$ & $\mathbf{7 . 3}$ & 85 & 86 & 149 & 148 \\
ship_001 & $\mathbf{1 3}$ & $\mathbf{1 3}$ & 140 & 146 & 312 & 318 \\
ship_003 & 404 & $\mathbf{3 0 9}$ & 5663 & 4543 & 3557 & 3225 \\
shipsec1 & 1075 & $\mathbf{3 8 8}$ & 17138 & 5683 & 6316 & 3778 \\
shipsec5 & 544 & $\mathbf{4 3 1}$ & 7964 & 6139 & 5183 & 4563 \\
shipsec8 & 641 & $\mathbf{4 1 3}$ & 9011 & 6217 & 4187 & 3640 \\
srb1 & $\mathbf{1 2}$ & $\mathbf{1 2}$ & 114 & 120 & 342 & 394 \\
thread & 145 & $\mathbf{5 2}$ & 2271 & 727 & 1086 & 627 \\
trdheim & $\mathbf{1 . 1}$ & $\mathbf{1 . 1}$ & 6 & 6 & 60 & 62 \\
troll & $\mathbf{3 4 6 8}$ & $\mathbf{3 6 1 2}$ & 54610 & 55210 & 13428 & 13433 \\
tsyl201 & $\mathbf{9 . 3}$ & $\mathbf{9 . 3}$ & 104 & 108 & 205 & 210 \\
tubu & $\mathbf{8 . 6}$ & $\mathbf{8 . 4}$ & 91 & 89 & 208 & 208 \\
x104 & $\mathbf{1 4 9}$ & $\mathbf{1 4 5}$ & 2054 & 2036 & 1882 & 1861 \\
\hline
\end{tabular}

parameters (with a minimum pivot block size of 16) and direct access files are used to store the matrix factors. In Table 4.6 timings for factorizing and solving for a single right-hand side are given, together with flop counts (the number of floating point operations required to factorize the matrix) and the number of entries in the matrix factors. For each problem, the fastest time is highlighted in bold (no distinction is made between the two times if the difference between them is less than 5 per cent). We see that the reductions in the wavefronts reported on in Table 4.3 lead to sparser factors, smaller flop counts, and substantial savings in the time required by the frontal solver MA42. For problems fcondp2 and shipsec1 the time is reduced by more than half. It is clear that, in general, the faster factorization times more than compensate for the extra time required to reorder the elements using the hybrid algorithm (see Table 4.4).

\section{Concluding remarks}

In this report, we have looked at using multilevel variants of Sloan's algorithm to reorder finiteelements for use with a frontal solver. Both direct and indirect versions of the reordering algorithm have been considered and used in combination with spectral orderings and the Hager exchange 
algorithm. Numerical experimentation showed that, in general, the best orderings are obtained using the indirect hybrid spectral-Sloan algorithm. We are currently developing a new Fortran 95 frontal solver for inclusion in the software library HSL (2004). Previous frontal solvers within HSL have required the user to preorder the elements but because using a good ordering is essential for the efficiency of the method, the new package will automatically reorder the elements for the user. Based on the results presented in this paper, the default setting will be to reorder the elements using the indirect hybrid spectral-Sloan algorithm, with the multilevel Fiedler code HSL MC73 called internally to compute the necessary spectral ordering. Because the MC63 Sloan orderings are fast and generally produce orderings of a similar quality for relatively small problems, the new package will include an option to reorder using MC63.

\section{Acknowledgements}

I would like to thank Ron Fowler of the Rutherford Appleton Laboratory and Christian Damhaug of Det Norske Veritas, Norway for supplying test problems.

\section{References}

S.T. Barnard and H.D. Simon. A fast multilevel implmentation of recursive spectral bisection for partitioning unstructured problems. Concurrency: Practice and Experience, 6, 101-117, 1994.

S.T. Barnard, A. Pothen, and H.D. Simon. A spectral algorithm for envelope reduction of sparse matrices. Numerical Linear Algebra with Applications, 2, 317-198, 1995.

I.S. Duff and J.A. Scott. The design of a new frontal code for solving sparse unsymmetric systems. ACM Trans. Mathematical Software, 22(1), 30-45, 1996.

I.S. Duff, J.K. Reid, and J.A. Scott. The use of profile reduction algorithms with a frontal code. Inter. Journal on Numerical Methods in Engineering, 28, 2555-2568, 1989.

M. Fiedler. A property of eigenvectors of nonnegative symmetric matrices and its application to graph theory. Czechoslovak Math. J., 25, 619-633, 1975.

W.W. Hager. Minimizing the profile of a matrix. SIAM J. Scientific Computing, 23(5), 17991816, 2002.

HSL. A collection of Fortran codes for large-scale scientific computation, 2004. See http://hsl.rl.ac.uk/.

Y.F. Hu and J.A. Scott. A multilevel algorithm for wavefront reduction. SIAM J. Scientific Computing, 23, 1352-1375, 2001.

Y.F. Hu and J.A. Scott. Ordering techniques for singly bordered block diagonal forms for unsymmetric parallel sparse direct solvers. Technical Report RAL-TR-2003-020, Rutherford Appleton Laboratory, 2003. 
G. Kumfert and A. Pothen. Two improved algorithms for envelope and wavefront reduction. BIT, 37:3, 559-590, 1997.

J.K. Reid and J.A. Scott. Ordering symmetric sparse matrices for small profile and wavefront. Inter. Journal on Numerical Methods in Engineering, 45, 1737-1755, 1999.

J.K. Reid and J.A. Scott. Implementing Hager's exchange methods for matrix profile reduction. ACM Trans. Mathematical Software, 28, 1-15, 2002.

J.A. Scott. On ordering elements for a frontal solver. Communications in Numerical Methods in Engineering, 15, 309-323, 1999.

S.W. Sloan. An algorithm for profile and wavefront reduction of sparse matrices. Inter. Journal on Numerical Methods in Engineering, 23, 1315-1324, 1986. 